\title{
Blockade of interleukin-6 signaling enhances hepatic steatosis but improves liver injury in methionine choline-deficient diet-fed mice
}

\author{
Kanji Yamaguchi ${ }^{1}$, Yoshito Itoh ${ }^{1}$, Chihiro Yokomizo ${ }^{1}$, Takeshi Nishimura ${ }^{1}$, Toshihisa Niimi ${ }^{1}$, Hideki Fujii ${ }^{1}$, \\ Takeshi Okanoue ${ }^{1,2}$ and Toshikazu Yoshikawa ${ }^{1}$
}

Inflammatory processes have an important role in the development of hepatic steatosis and progression to nonalcoholic steatohepatitis (NASH). Interleukin-6 (IL-6) is known to be a proinflammatory cytokine, but also promotes liver regeneration and protects the liver against various forms of damage. The role of IL-6/Glycoprotein130 (GP130) in NASH remains unclear. In this study, we determined whether blocking IL-6/GP130 signaling prevents progression of steatohepatitis in a mouse NASH model. Six-week-old male C57/BL6 mice were fed either chow control or a methionine cholinedeficient (MCD) diet for 8 weeks. Half of the MCD diet-fed mice were treated with $15 \mathrm{mg} / \mathrm{kg}$ rat anti-mouse IL-6 receptor antibody (MR16-1), intraperitoneally twice weekly, the remainder and chow-fed mice were injected with $15 \mathrm{mg} / \mathrm{kg}$ rat $\mathrm{lgG}$ as a control. Hepatic steatosis, injury, fibrosis, apoptosis, markers of lipid peroxidation/oxidant stress and IL-6-related gene expressions were evaluated. MR16-1 treatment decreased signal transducer and activator of transcription 3 activities and expression of suppressor of cytokine signaling 3 in MCD diet-treated mouse livers. Although this treatment enhanced intrahepatic lipid accumulation accompanied by increased sterol regulatory element-binding protein 1 and decreased peroxisome proliferator-activated receptor- $\alpha$ expression, elevated plasma alanine aminotransferase levels were improved with decreased plasma free fatty acid levels, lipid peroxidation/oxidant stress and hepatic apoptosis. Blocking IL-6/GP130 signaling by MR16-1 enhanced MCD diet-induced hepatic steatosis, but ameliorated liver injury. These findings suggest that hepatic IL- 6 signaling has a protective role against the progression of hepatic steatosis but may enhance liver inflammation.

Laboratory Investigation (2010) 90, 1169-1178; doi:10.1038/labinvest.2010.75; published online 5 April 2010

KEYWORDS: NASH; IL-6; MCD diet; STAT3; SREBP-1

Nonalcoholic fatty liver disease (NAFLD) is one of the most common liver diseases worldwide. ${ }^{1}$ According to the two-hit hypothesis for NAFLD progression, hepatic steatosis is a risk factor for nonalcoholic steatohepatitis (NASH) and fibrosis. ${ }^{2}$ Continuous excess triglyceride accumulation induces proinflammatory cytokines, produces reactive oxygen species (ROS) and leads to NASH. Proinflammatory cytokines, such as tumor necrosis factor-alpha (TNF- $\alpha$ ) and interleukin (IL)-6, are believed to have a central role in this process. Plasma TNF- $\alpha$ levels have been reported to be higher in patients with NASH than in patients with steatosis and controls with no known liver diseases. ${ }^{3,4}$ In vivo studies have shown that treatment of leptin-deficient ob/ob mice with antibodies against TNF- $\alpha$ improved NASH and hepatic insulin resistance. Mice genetically deficient in the TNF type 1 receptor were resistant to steatosis and liver injury induced by a highcarbohydrate and a methionine choline-deficient (MCD) diet. $^{5}$

Although cytokine imbalance and, in particular, an increase in TNF- $\alpha$ may have a key role in the development of $\mathrm{NASH}$, the role of IL-6 remains obscure. IL- 6 is a cytokine with numerous effects, which are dependent on TNF- $\alpha$ acting as an upstream mediator. ${ }^{6,7}$ Previous studies have shown that, despite no significant difference between fatty liver and NASH patients, inflammatory C-reactive protein (CRP) was increased in both liver and adipose tissues in patients with

\footnotetext{
${ }^{1}$ Molecular Gastroenterology and Hepatology, Graduate School of Medical Science, Kyoto Prefectural University of Medicine, Kyoto, Japan and ${ }^{2}$ Center of Gastroenterology and Hepatology, Saiseikai Suita Hospital, Osaka, Japan

Correspondence: Dr Y Itoh, MD, PhD, Department of Molecular Gastroenterology, Kyoto Prefectural University of Medicine, 465 Kajii-cho, Kawaramachi-Hirokoji, Kamigyou-ku, Kyoto 602-8566, Japan.

E-mail: yitoh@koto.kpu-m.ac.jp

Received 28 July 2009; revised 23 December 2009; accepted 11 February 2010
} 
severe obesity. Basically, CRP mRNA levels are correlated positively with those of IL- 6 and the liver, and also adipose tissue, and can produce CRP, a process which could be dependent on IL- $6 .{ }^{8}$ These findings suggest a role for IL- 6 as a proinflammatory cytokine in the development of NASH. Moreover, latest reports have shown that hepatocyte IL-6 expression correlated positively with plasma IL-6 levels and the degree of hepatic inflammation, stage of fibrosis and systemic insulin resistance. ${ }^{3,4,9}$

From a different point of view, IL-6 is well known to be a hepato-protective cytokine. IL-6 acts directly on hepatocytes, inducing the translocation of signal transducer and activator of transcription (STAT) 3 to the nucleus causing early gene activation and mitosis. ${ }^{10,11}$ The IL-6/soluble IL-6 receptor fusion protein led to an earlier onset of hepatocellular proliferation and had an important role in liver regeneration after partial hepatectomy in mice. ${ }^{12}$ This signaling not only has an effect on hepatocyte proliferation but also protects the liver against various forms of injury, such as ischemia and reperfusion, toxins, alcohol and death-mediated Fas activation. ${ }^{13-15}$ Mice lacking IL-6 have an impaired ability to regenerate their livers after partial hepatectomy, increased caspase 3/8 activities and reduced antiapoptotic mediators. ${ }^{6,14,16,17}$ Short-term replacement of IL-6 also might protect the liver through preservation and restoration of intrahepatic adenosine triphosphate (ATP) levels, which have been reported to be reduced in fatty livers. ${ }^{18}$

Chronic inflammation is a risk for the development of fibrosis and carcinogenesis in various organs. ${ }^{19,20}$ Controlling the inflammation would prevent the progression of this process, even if it may not be able to stop the occurrence of the diseases altogether. Recently, inhibition of the proinflammatory cytokine signaling pathway using neutralizing antibody against the IL-6 receptor (tocilizumab, which is a specific antagonist of the human IL-6 receptor and soluble IL- 6 receptor) has been reported to improve chronic inflammatory diseases, such as rheumatoid arthritis, in clinical practice. ${ }^{21}$ In this study, to evaluate the possible role of IL-6 in the development of hepatic steatosis and $\mathrm{NASH}$, we tested a rat anti-mouse IL-6 receptor antibody, MR16-1, a specific antagonist of the mouse IL-6 receptor, in mouse NASH models. Feeding mice with an MCD diet induces steatohepatitis and liver fibrosis, providing a useful small animal model for $\mathrm{NASH} .^{22}$ Using this model, we found that, although treatment with MR16-1 enhanced hepatic steatosis, it ameliorated liver injury. Neutralizing the IL-6 receptor by MR16-1 for 8 weeks suppressed IL-6/ Glycoprotein130 (GP130) signaling successfully and decreased expression of suppressor of cytokine signaling (SOCS) 3 in correlation with decreased plasma free fatty acid (FFA) levels, hepatic Cyp2E1 expression, evidence of lipid peroxidation/oxidant stress and hepatocyte apoptosis. In conclusion, inhibiting IL-6/GP130 signaling enhanced hepatic steatosis, but ameliorated liver damage in mice with MCD diet-induced NASH.

\section{MATERIALS AND METHODS}

\section{Animals and Treatments}

A mouse model of MCD diet-induced NASH was studied. Six-week-old male C57/BL6 mice were purchased from Japan Jackson Laboratories, maintained in a temperature- and light-controlled facility and permitted consumption of water ad libitum. In all, 5 mice were fed a control chow diet (cat no. 960441; ICN, Aurora, OH) and 10 were fed an MCD diet (cat no. 960439; ICN) for 8 weeks. Half of the MCD diet-fed mice $(N=5)$ were treated with $15 \mathrm{mg} / \mathrm{kg}$ rat anti-mouse IL-6 receptor antibody (MR16-1; Cyugai Pharmaceutical, Tokyo, Japan) intraperitoneally twice weekly, the remainder $(N=5)$ and five chow-fed mice were injected with control rat $\operatorname{IgG}$ (Equitech-Bio, Kerrville, TX). ${ }^{23,24}$ All animal experiments fulfilled the requirements for humane animal care in the Kyoto Prefectural University of Medicine.

\section{Immunoblot Assay}

Nuclear proteins isolated using a NE-PER Nuclear Extraction Reagent Kit (Pierce Biotechnology, Rockford, IL) and proteins isolated from whole livers were electrophoresed in SDS-PAGE gels and transferred to PVDF membranes. The membranes were probed with anti-sterol regulatory element-binding protein (SREBP)-1 (Santa Cruz Biotechnology, sc-366 for the precursor and sc-367 for the mature fragment, Santa Cruz, CA), anti-peroxisome proliferator-activated receptor (PPAR) $\alpha$ (LifeSpan Biosciences, WA), anti-4-hydroxy-2-nonenal (HNE) (HNEJ-2; Nikken, Shizuoka, Japan), anti-cytochrome P4502E1 (Cyp2E1) (Stressgen, Victoria, Canada), anti-catalase (Abcam, Cambridge, MA) anti-STAT3, anti-phospho-STAT3, anti-Akt, anti-phospho-Akt (ser473), anti-adenosine monophosphateactivated protein kinase (AMPK), anti-phospho-AMPK (Thr172), (Cell Signaling Technology, Beverly, MA) antibody, anti- $\alpha$-actin (Santa Cruz Biotechnology) antibody or antiglyceraldehydes-3-phosphate dehydrogenase (GAPDH) (Santa Cruz Biotechnology) antibody, followed by horseradish peroxidase (HRP)-conjugated anti-mouse or rabbit IgG (Amersham, UK). Antigens were visualized by ECL (Amersham). Immunoblots were scanned, and band intensities were quantified by Image J (NIH) densitometry analysis.

\section{Two-Step Real-Time RT-PCR}

Real-time PCR was performed as described previously. ${ }^{25}$ Specificity was confirmed for all primer pairs (Table 1) by sequencing the PCR products. Target gene levels are presented as a ratio of levels in treated $v s$ corresponding control groups. Fold changes were determined using point and interval estimates.

\section{Immunohistochemistry and Analysis of Liver Architecture}

Serial sections were stained with H\&E using standard techniques. After deparaffinization, microwave antigen retrieval and blocking endogenous peroxidase activity, other sections were incubated with a TdT-mediated dUTP-digoxigenin 
nick-end labeling (TUNEL) reaction mixture, containing terminal deoxyribonucleotidyl transferase (TdT) and fluorescein-dUTP (Roche Diagnostic, Indianapolis, IN), or anti- $\alpha$ smooth muscle actin (SMA) (DakoCytomation, Carpinteria, CA) antibody. Antigen was demonstrated using secondary anti-mouse polymer HRP and DAB chromagen (DakoCytomation) and counterstaining with Gill's hematoxylin. The number of TUNEL-positive hepatocytes was counted in three randomly selected fields per section ( $\times 100$ magnification).

Table 1 RT-PCR primers for analysis

\begin{tabular}{lll}
\hline Gene & Direction & Sequence \\
\hline Gus & Forward & GCAGTGGGTGGGTGATGG \\
& Reverse & GGGTCAGTGTGTGTGATGG \\
SOCS3 & Forward & GGGTGGCAAAGAAAAGGA \\
& Reverse & GTGAGCGTCAAGACCCAGT \\
TNF $\alpha$ & Forward & TCGTAGCAAACCACCAAGTG \\
& Reverse & AGATAGCAAATCGGCTGACG \\
TGF $\beta$-1 & Forward & TGGCCTCTACAACCAACACAA \\
& Reverse & GGCTTGCGACCCACGTAGTA \\
Pro Col1 $\alpha 1$ & Forward & GACATCCCTGAAGTCAGCTGC \\
& Reverse & TCCCTTGGGTCCTCGAC \\
$\alpha$-SMA & Forward & AAACAGGAATACGACGAAG \\
& Reverse & CAGGAATGATTGGAAAGGA \\
FATP1 & Forward & CGCTITCTGCGTATCGTCTG \\
& Reverse & GATGCACGGGATCGTGTCT \\
FATP2 & Forward & GGTATGGGACAGGCCTTGCT \\
& Reverse & GGGCATGTGGTATAGATGACATC \\
FATP5 & Forward & CTACGCTGGCTGCATATAGATG \\
& Reverse & CCACAAAGGTCTCTGGAGGAT \\
\hline
\end{tabular}
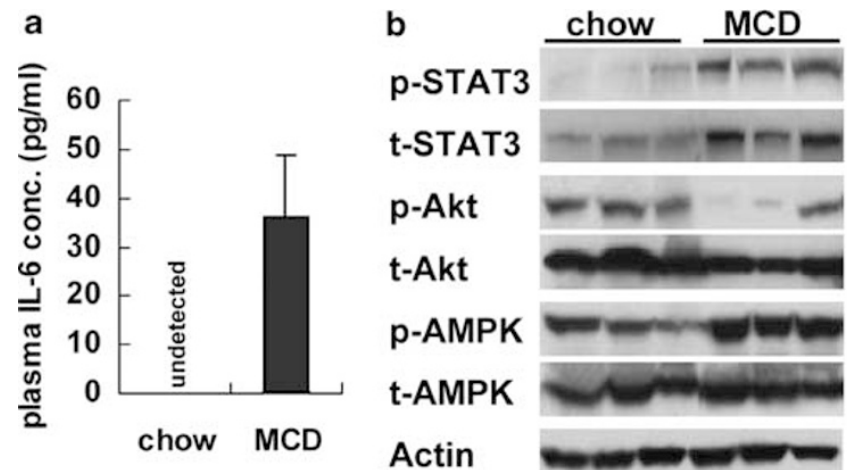

C



\section{Quantification of Hepatic Collagen Content}

Sirius red staining was performed on $4-\mu$ m-thick paraffin sections of liver tissues fixed in buffered formalin. After deparaffinization, the sections were treated with $0.1 \%$ Sirius Red F3B (Sigma-Aldrich, Japan) and 0.1\% Fast Green FCF (Sigma-Aldrich) in saturated Picric acid solution (SigmaAldrich) for $30 \mathrm{~min}$. Sirius red staining area was quantitated by Image $J$ software in three randomly selected fields per section ( $\times 200$ magnification).

\section{Tissue and Plasma Biochemical Measurements}

Plasma alanine aminotransferase (ALT), FFA, total cholesterol, triglyceride and glucose levels were measured as described previously. ${ }^{25}$ Plasma IL-6, serum amyloid A (SAA), TNF- $\alpha$ concentrations and tissue triglyceride were measured using an IL-6 Mouse ELISA Kit (R\&D Systems, Minneapolis, MN), SAA mouse ELISA kit (Stressgen), TNF- $\alpha$ mouse ELISA Kit (Invitrogen, Japan) and Triglyceride Detection Kit (Sigma-Aldrich) according to the manufacturers' instructions.

\section{Statistical Analysis}

Results are expressed as mean \pm s.e.m. Significance was established using Student's $t$-test and analysis of variance when appropriate. Differences were considered significant when $P<0.05$.

\section{RESULTS}

An MCD Diet Elevated Plasma IL-6 Levels and Activated Hepatic STAT3 Signaling

We measured plasma IL-6 levels by ELISA in MCD diet-fed mice and compared them with those in chow diet-fed mice. As in previous studies, ${ }^{26,27}$ an MCD diet increased IL-6 levels dramatically (Figure 1a) and also activated the expression of a key gene in IL-6/GP130 signaling, STAT3 (Figure 1b). Plasma SAA levels were also increased in MCD diet-fed mice

Figure 1 The effect of an MCD diet on plasma IL-6 and SAA levels and hepatic IL-6/ GP130-related gene expression. (a) Plasma IL-6 levels were measured. Mean \pm s.e. data from each group ( $n=5$ per group) are plotted at 8 weeks. (b) Phosphorylated (p)-stat3, total (t)-STAT3, p-Akt, t-Akt, p-AMPK and t-AMPK levels were evaluated by immunoblot analysis of livers obtained from three mice per group after 8 weeks. To control for loading, the blot was stripped and reprobed for $\beta$-actin, a housekeeping gene. (c) Plasma SAA levels were measured. Mean \pm s.e. data from each group $(n=5$ per group) are plotted at 8 weeks $\left({ }^{*}<<0.05\right)$. (d) mRNA levels of SOCS3 were determined by quantitative real-time PCR analysis of total liver RNA obtained after 8 weeks. Results were normalized to glucuronidase (GUS) expression in each sample and then expressed as fold change relative to gene expression in chow-fed control mice. Mean \pm s.e. data from each group ( $n=5$ per group) $(P=0.094)$. 

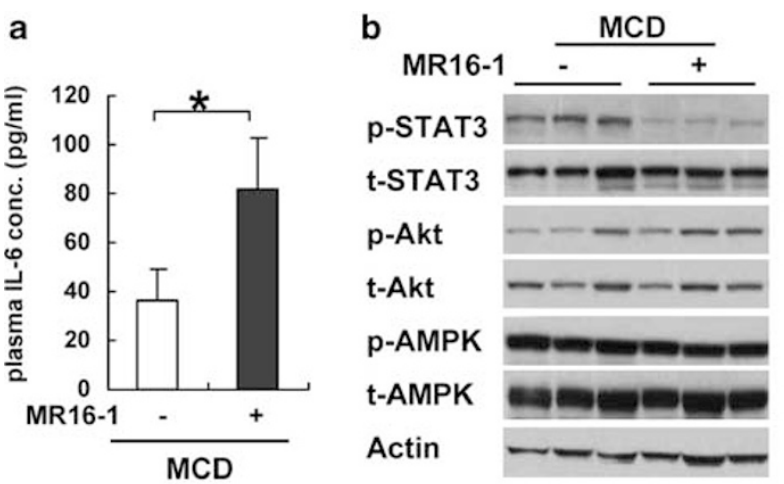

t-STAT3

p-Akt
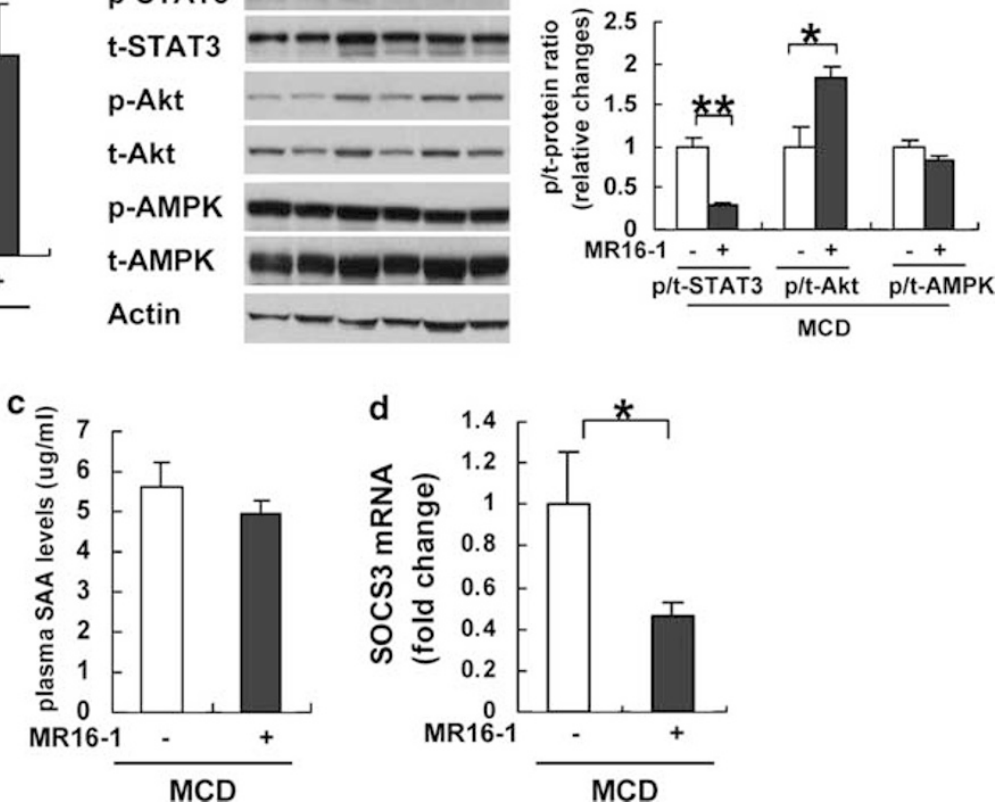

Figure 2 The effect of MR16-1 treatment on plasma IL-6 levels and hepatic IL-6/GP130-related gene expression in MCD diet-fed mice. (a) Plasma IL-6 levels were measured. Mean \pm s.e. data from each MCD diet-fed group ( $n=5$ per group) are plotted at 8 weeks. (b) P-STAT3, STAT3, p-Akt, Akt, p-AMPK and AMPK levels were evaluated by immunoblot analysis of livers obtained from three mice per group after 8 weeks. To control for loading, the blot was stripped and reprobed for $\beta$-actin. P-STAT3/t-STAT3, P-Akt/t-Akt and p-AMPK/t-AMPK ratios in each group were also expressed as fold change of control IgG-treated mice $\left({ }^{*} P<0.05\right)$. (c) Plasma SAA levels were measured. Mean \pm s.e. data from each group ( $n=5$ per group) are plotted at 8 weeks. (d) mRNA levels of hepatic SOCS3 were determined by quantitative real-time PCR analysis after 8 weeks of treatment. Results were normalized to GUS expression and then expressed as fold change relative to gene expression in controls. Mean \pm s.e. data from each MCD diet-fed group ( $n=5$ per group) ( ${ }^{*}<0.05$ ).

(Figure 1c). The expression of genes involved in glucogenesis and lipogenesis, Akt and AMPK, was also assessed. An MCD diet induced AMPK phosphorylation but reduced Akt phosphorylation (Figure 1b). Furthermore, mRNA levels of hepatic SOCS3 were slightly (but not significantly, $P=0.094$ ) increased in MCD mice (Figure 1d), suggesting that activation of STAT3 by an MCD diet might inhibit Akt phosphorylation through induction of SOCS3.

\section{Neutralizing the IL-6 Receptor Blocked IL-6/GP130 Signaling}

In this study, we used MR16-1 to block the IL-6/GP130 pathway because earlier studies had shown that twice weekly intraperitoneal injections of $15 \mathrm{mg} / \mathrm{kg}$ MR16-1 suppressed the IL6/GP130 pathway in mice effectively. ${ }^{24}$

First, to examine the efficacy of MR16-1, we measured plasma IL-6 concentrations after MR16-1 treatment, and found that plasma IL-6 levels were greatly increased. This phenomenon may be caused by decreased clearance of IL- 6 from the blood ${ }^{28}$ and suggests that MR16-1 treatment blocked the IL-6 receptor successfully (Figure 2a, Supplementary Figure 1A). This treatment had little effect on STAT3 phosphorylation in chow-fed controls but greatly decreased MCD diet-induced STAT3 phosphorylation (Figure 2b, Supplementary Figure 1B). This result showed that profound suppression of hepatic STAT3 activity might be attributed to the inhibition of IL-6 signaling by MR16-1 treatment in MCD diet-fed mice. Moreover, Akt phosphorylation was somewhat recovered by MR16-1 treatment in both chowand MCD diet-fed mice. In contrast, AMPK phosphorylation seems slightly decreased in MCD diet-fed mice (Figure 2b, Supplementary Figure 1B). MR16-1 treatment had no effect on plasma SAA levels in chow-fed mice, but slightly decreased plasma SAA levels in MCD diet-fed mice (Figure 2c, Supplementary Figure 1C). mRNA levels of hepatic SOCS3 expression were also decreased by $>50 \%$ in only MCD dietfed mice (Figure 2d, Supplementary Figure $1 \mathrm{D},{ }^{\star} P<0.05$ ).

\section{Neutralizing the IL-6 Receptor Exacerbated MCD Diet- Induced Hepatic Steatosis, but it Decreased Plasma FFA Levels}

As some reports have shown that the activation of STAT3 improved hepatic steatosis by inhibiting expression of SREBP-1, ${ }^{11,29}$ neutralizing the IL-6 receptor might be expected to exacerbate hepatic steatosis. This was evaluated by $\mathrm{H} \& \mathrm{E}$ staining. Steatosis was scored on H\&E-stained sections according to Brunt's criteria ${ }^{30}$ and photomicrographs from representative mice are shown (Figure 3a, Supplementary Figure 2A). An MCD diet for 8 weeks induced a mild diffuse hepatic steatosis compared with chow diet. Furthermore, 
a chow

MCD

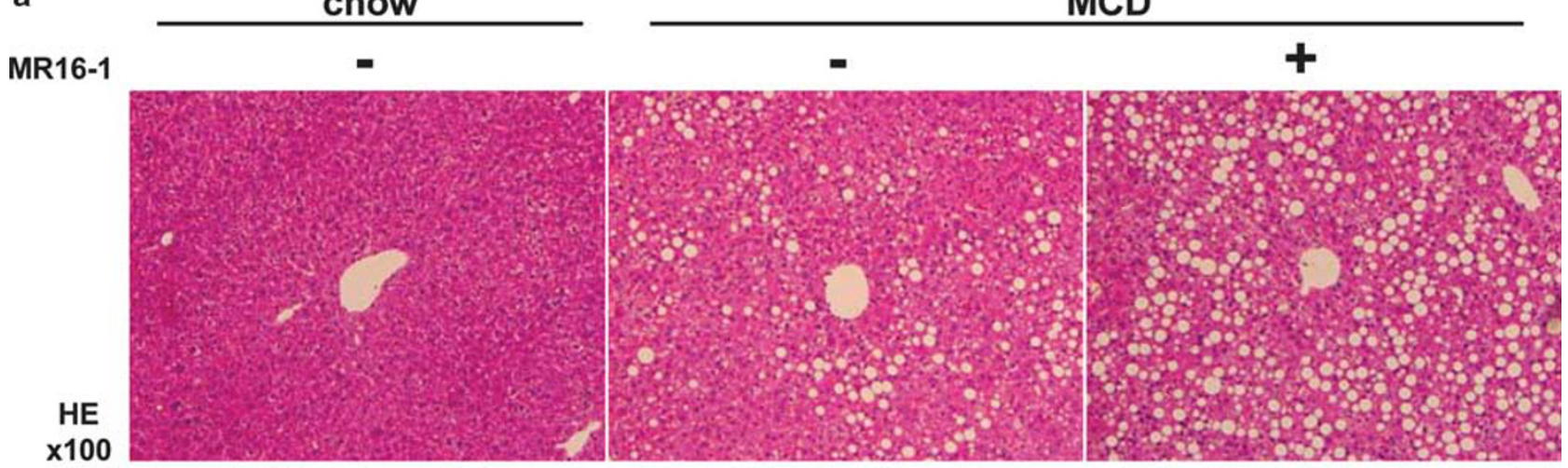

b (g)
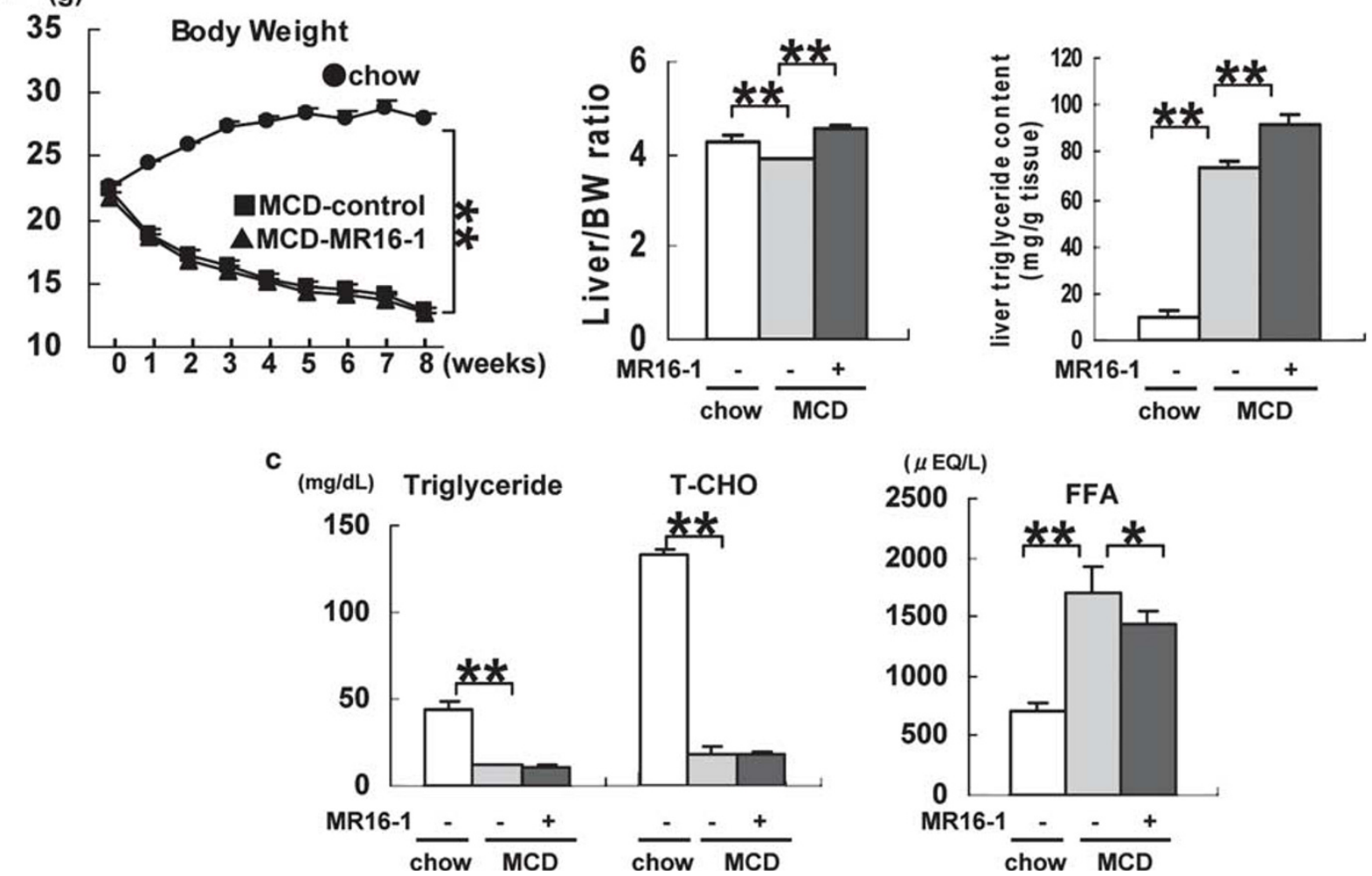

Figure 3 Liver histology, BW, liver/BW ratio, liver triglyceride content and plasma total-cholesterol, triglyceride and FFA levels in chow- or MCD diet-fed mice treated with control IgG or MR16-1. (a) Hematoxylin and eosin staining of liver sections from representative mice from each treatment group. Hepatic steatosis grade was assessed on sections obtained from each mouse. Mean \pm s.e. data from each group ( $n=5$ per group) are displayed $\left({ }^{*} P<0.01\right)$. (b) Body weight (BW) was assessed weekly until the end of the treatment. Liver/BW ratio and liver triglyceride content were determined at the end of the treatment. Results of liver triglycerides are expressed per gram tissue. Mean \pm s.e. data from each group are plotted $(* * P<0.01)$. (c) Plasma total-cholesterol (T-CHO), triglyceride and FFA levels were determined in each group ( $n=5$ per group) at the end of the 8-week treatment period. Data are presented as mean \pm s.e. $\left({ }^{*} P<0.05\right.$, $\left.{ }^{*} P<0.01\right)$. 
a

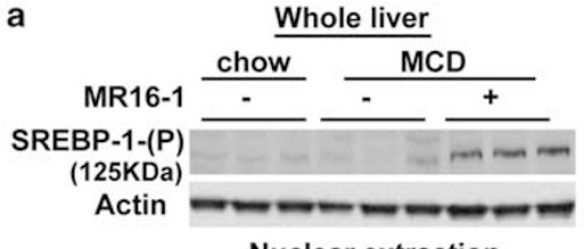

Nuclear extraction

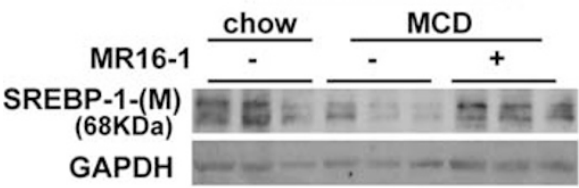

b

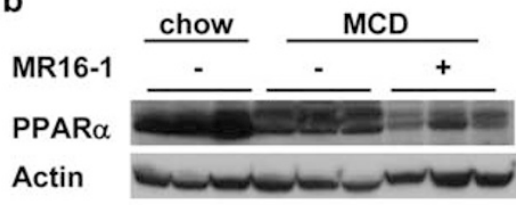

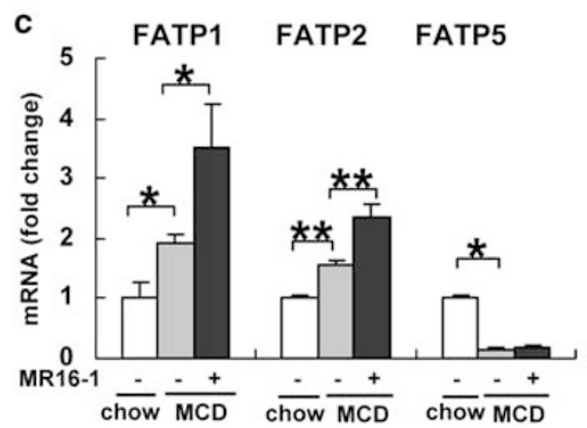

Figure 4 The effect of MR16-1 treatment on hepatic lipogenic and fatty acid uptake-related gene expression. (a) SREBP-1 precursor (P) (MW: $125 \mathrm{kDa}$ ) and mature soluble fragment $(\mathrm{M})(\mathrm{MW}: 68 \mathrm{kDa}$ ) levels were evaluated by immunoblot analysis of whole livers or nuclear extraction from three mice per group after 8 weeks. To control for loading, the blot was stripped and reprobed for $\beta$-actin or GAPDH, respectively. (b) PPAR $\alpha$ levels were evaluated by immunoblot analysis of livers from three mice per group after 8 weeks of treatment. To control for loading, the blot was stripped and reprobed for $\beta$-actin. (c) mRNA levels of hepatic FATP1, 2 and 5 were determined by quantitative real-time PCR analysis after 8 weeks of treatment. Results were normalized to GUS expression and then expressed as fold change relative to gene expression in chow-fed control mice. Mean \pm s.e. data from each group $(n=5$ per group) $\left({ }^{*} P<0.05,{ }^{*} P<0.01\right)$.

MCD diet-fed, but not chow-fed, mice treated with MR16-1 had a greater degree of hepatic steatosis than those treated with control IgG. These findings were confirmed by analysis of body weight (BW), liver/BW ratio and biochemical analysis of hepatic triglyceride content (Figure 3b, Supplementary Figure 2B). Interestingly, MR16-1 treatment attenuated the MCD diet-induced increases in plasma FFA without decreasing plasma triglyceride and total cholesterol levels (Figure 3c, Supplementary Figure 2C).

\section{Neutralizing the IL-6 Receptor Enhanced Hepatic Fatty Acid Synthesis and Uptake}

We assessed the protein levels of SREBP-1 expression to investigate their correlation with decreased STAT3 activity and exacerbated hepatic steatosis after MR16-1 treatment. An MCD diet had no effect on the SREBP-1 precursor (molecular weight: $125 \mathrm{kDa}$ ) that is attached to the nuclear envelope and endoplasmic reticulum but slightly decreased the mature soluble fragment (molecular weight: $68 \mathrm{kDa}$ ) in the nucleus (Figure 4a). MR16-1 treatment increased both forms of SREBP-1 in MCD diet-fed mouse livers (Figure 4a). An MCD diet decreased the protein levels of PPAR $\alpha$, and MR16-1 treatment further accelerated the reduction (Figure $4 \mathrm{~b}$ ). In our study, although the fatty acid transport protein (FATP) 3, 4 (data not shown) and 5 mRNA levels were not altered, FATP 1 and 2 were significantly upregulated in MCD diet-fed mice (but not significantly in chow-fed mice) by MR16-1 treatment (Figure $4 \mathrm{c},{ }^{\star} P<0.05$, Supplementary Figure $3 \mathrm{C}$ ).

\section{Neutralizing the IL-6 Receptor had no Effect on MCD Diet-Induced Liver Fibrosis}

Hepatic steatosis has been identified as a risk factor for liver fibrosis. Therefore, we assessed liver fibrosis by real-time PCR of mRNA levels of various markers of fibrosis. Although an MCD diet significantly increased mRNA levels of hepatic collagen and transforming growth factor $\beta 1$ expression, compared with chow control, MR16-1 treatment had no effect on those levels (Figure $5 \mathrm{a},{ }^{\star} P<0.05$ ). Sirius red staining and $\alpha$-SMA immunohistochemistry were performed to further assess the effects of MCD diet and MR16-1 treatment on liver fibrosis. However, MR16-1 treatment had no effect on the area of Sirius red-stained fibrils and $\alpha$-SMA staining cells (Figure 5b).

\section{Neutralizing the IL-6 Receptor Ameliorated MCD Diet- Induced Liver Injury}

To evaluate liver injury after MR16-1 treatment, we compared injury-related parameters. As expected, MCD diet-fed mice exhibited 10-fold higher plasma ALT values than did the controls (Figure 6a). However, plasma ALT values were 

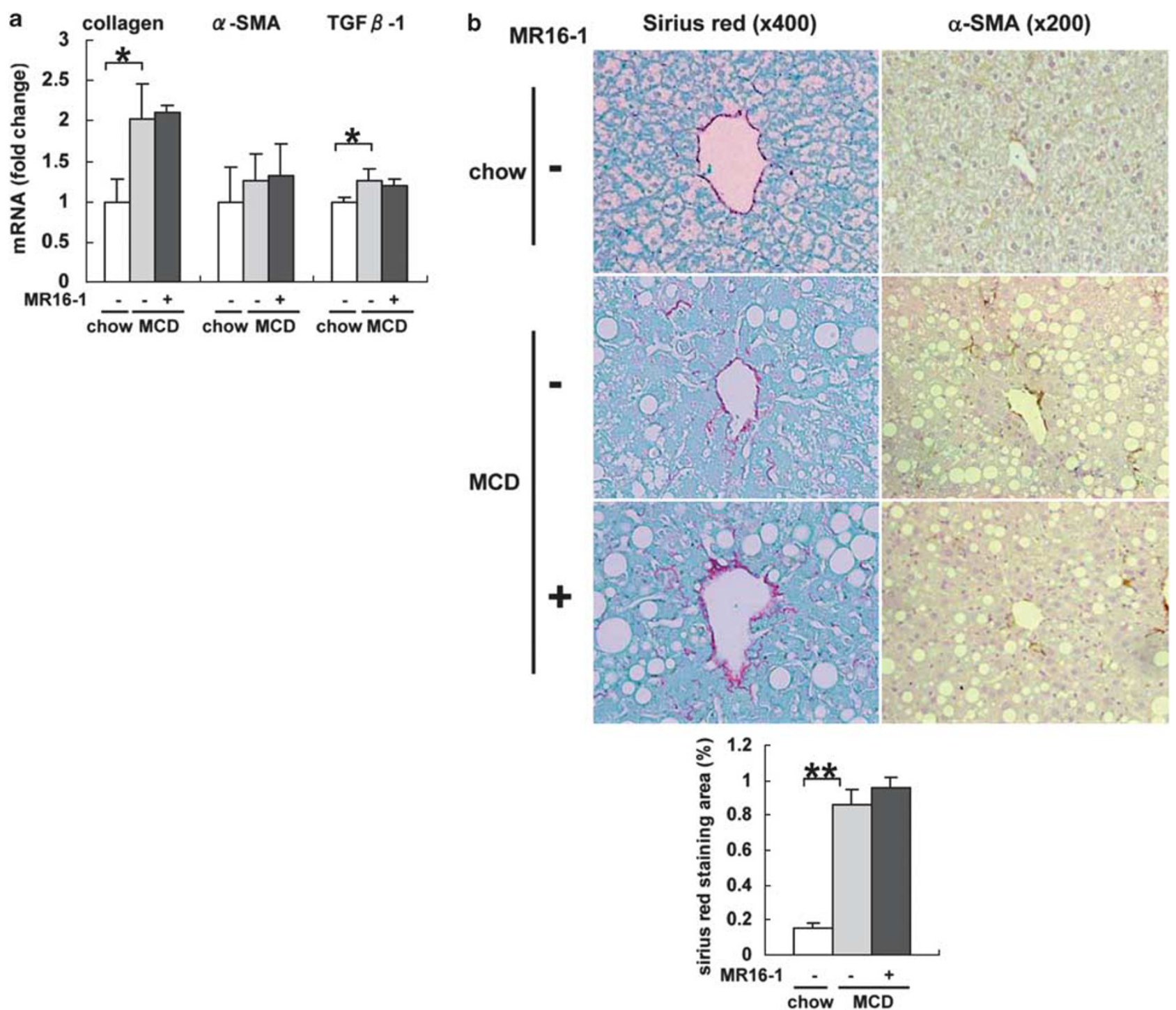

Figure 5 Markers of fibrosis in chow- or MCD diet-fed mice treated by control IgG or MR16-1. (a) mRNA levels of hepatic collagen, $\alpha$-SMA and TGF $\beta-1$ were determined by quantitative real-time PCR analysis after 8 weeks of treatment. Results were normalized to GUS expression. Mean \pm s.e. data from each sample are displayed as fold change relative to chow-fed control mice $\left({ }^{*} P<0.05\right)$. (b) Liver sections from all mice were stained with Sirius red and $\alpha$-SMA antibody after 8 weeks of treatment. Photomicrographs from representative sections are shown. Morphometric analysis of Sirius red-stained sections from each MCD diet-fed group ( $n=5$ per group) at 8 weeks. Results are expressed as $\%$ of section staining $(+)$ for Sirius red.

significantly lower in MR16-1-treated mice than in control IgG treated-mice (Figure $6 \mathrm{a},{ }^{*} P<0.01$ ). These observations were seen only in MCD diet-fed mice (Supplementary Figure $3 \mathrm{~A}$ ). In many mouse models of fatty liver diseases and insulin resistance, as well as in humans with similar conditions, TNF- $\alpha$ has been implicated to have an important role in the pathogenesis of NASH. In this study, however, MR16-1 treatment had little effect on MCD diet-induced elevated plasma TNF- $\alpha$ levels and hepatic TNF- $\alpha$ mRNA expression (Figure 6b, Supplementary Figure 3B). Liver sections were also evaluated for lobular inflammation. The lobular inflammatory grade was higher in MCD diet-fed mice than in chow-fed controls. MR16-1 treatment slightly ameliorated liver injury in MCD diet-fed mice (Figure 6c). To determine why neutralizing the IL-6 receptor in MCD diet-fed mice ameliorated liver injury but exacerbated liver steatosis, we compared the expression of microsomal and peroxisomal FFA-oxidizing enzymes and markers of lipotoxicity. MR16-1 treatment decreased protein levels of hepatic Cyp2E1, catalase and MCD diet-induced 4-HNE expression (Figure 6d). Furthermore, TUNEL staining also showed that MCD dietinduced hepatic apoptosis was decreased by MR16-1 treatment (Figure 6e).

\section{DISCUSSION}

According to the 'two-hit' hypothesis, oxidative stress, lymphocyte activation and cytokine release are candidate elements of the pathogenic transition from simple steatosis to 



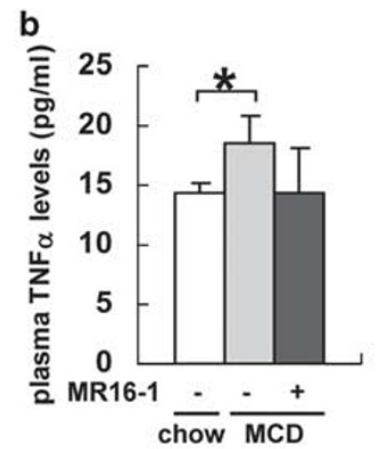

e

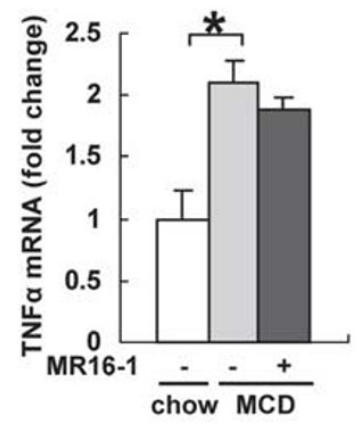

TUNEL
C

\begin{tabular}{|c|c|}
\hline & $\begin{array}{c}\text { inflammatory } \\
\text { score }\end{array}$ \\
\hline Chow+control & $0.2( \pm 0.2)$ \\
\hline MCD+control & $0.9( \pm 0.12)$ \\
\hline MCD+MR16-1 & $0.7( \pm 0.13)$ \\
\hline \multicolumn{2}{|c|}{$\begin{array}{l}0: \text { no inflammatory foci per } 20 x \text { field } \\
1: 1-2 \text { inflammatory foci per } 20 x \text { field } \\
2: 3-4 \text { inflammatory foci per } 20 x \text { field } \\
3:>4 \text { inflammatory foci per } 20 x \text { field }\end{array}$} \\
\hline
\end{tabular}
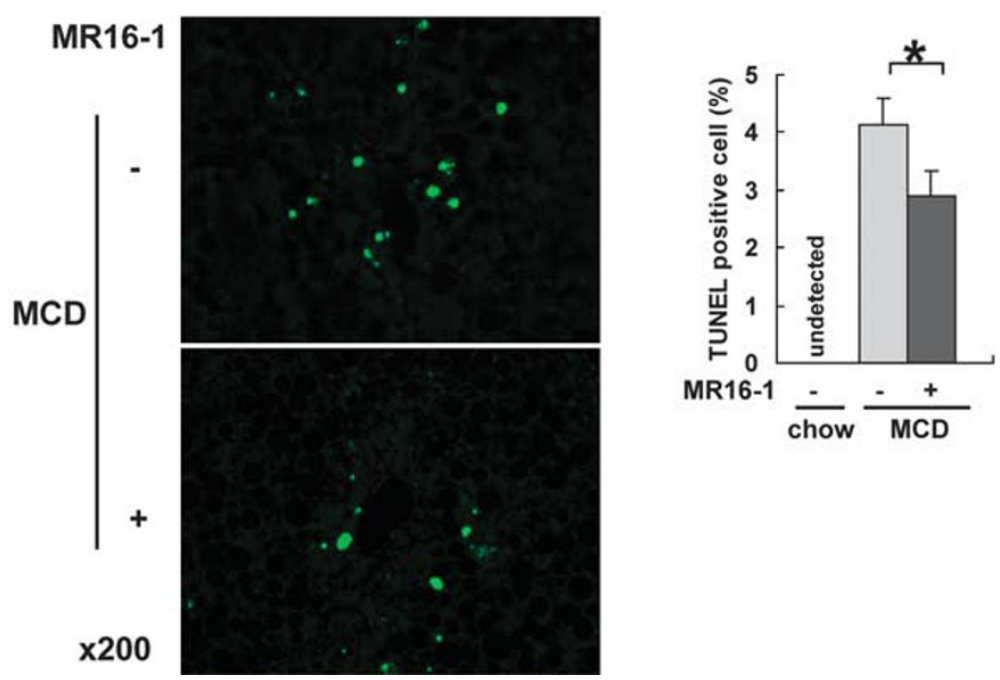

Figure 6 Injury-, lipotoxicity- and apoptosis-related parameters in chow- or MCD diet-fed mice treated by control IgG or MR16-1. (a) Plasma ALT levels were measured after 8 weeks of treatment. Mean \pm s.e. results from each group ( $n=5$ per group) $\left.{ }^{* *} P<0.01\right)$. (b) Plasma TNF- $\alpha$ levels were measured from each group ( $n=5$ per group) at 8 weeks. The mRNA levels of hepatic TNF- $\alpha$ were also evaluated by quantitative real-time PCR analysis. Results were normalized to GUS expression. Mean \pm s.e. data are relative to chow-fed control mice $\left({ }^{*} P<0.05\right)$. (c) The number of inflammatory foci per $\times 20$ field was counted on sections obtained from each mouse. Mean \pm s.e. data from each group ( $n=5$ per group) are displayed $(* * P<0.01)$. (d) Cyp2E1, catalase and 4-HNE levels were evaluated by immunoblot analysis of livers from three mice per group after 8 weeks. To control for loading, the blot was stripped and reprobed for $\beta$-actin. (e) Apoptosis of hepatocytes was evaluated by TUNEL staining of liver sections after 8 weeks of treatment. Photomicrographs from representative mice are shown. Morphometric analysis of TUNEL-stained sections from each MCD diet-fed group ( $n=5$ per group) at 8 weeks. Results are expressed as $\%$ of nuclei staining $(+)$ for TUNEL $(* P<0.05)$.

steatohepatitis. $^{2}$ We focused on the role of IL-6 as a proinflammatory cytokine and used an inhibitor of the IL-6 receptor, MR16-1, in an MCD diet-induced NASH model, because an MCD diet was found to increase plasma IL-6 levels significantly and to activate hepatic IL6/GP130-related gene expression. Consequently, treatment of these mice with MR16-1 enhanced hepatic steatosis but ameliorated liver cell injury.

These contrasting observations regarding hepatic steatosis and inflammation indicate a paradoxical role for IL-6 in the development of chronic steatosis and inflammation of the liver. Recently, Horiguchi et al reported that, although STAT3 in hepatocytes, which depends on IL-6, could contribute to promote liver inflammation but inhibit steatosis by inhibiting SREBP-1 expression directly, STAT3 in macrophages/ Kupffer cells, which depends on IL-10, could suppress liver inflammation during alcoholic liver injury. ${ }^{29}$ This report reinforces our results. In our study, MR16-1 treatment blocked the MCD diet-induced hepatic STAT3 activation (Figure 2b) and ameliorated liver cell injury (Figure 6a). Interestingly, MR16-1 treatment also induced greater hepatic steatosis accompanied by increased hepatic SRBP-1 expression (Figures 3a and 4a). Further study regarding STAT3 activation in isolated hepatic macrophages/Kupffer cells obtained from MCD-diet fed mice and the effects of IL-10 is recommended in the future.

The role of IL- 6 in the pathogenesis of NAFLD has been an issue of debate. Although IL-6 is well known as one of several proinflammatory cytokines active during infection, cahexia and obesity, IL-6 also is protective against various forms of liver damage. ${ }^{4-7,10-17}$ According to the paper by Wallenius et $a{ }^{31}$ IL-6-deficient mice develop mature-onset obesity, which is reversed by IL-6 replacement. This finding is interesting and also reinforces our data that blocking IL-6/GP130 
signaling deteriorates hepatic steatosis in MCD-diet induced NASH model. Therefore, the therapeutic use of IL-6/GP130 signaling in treating obesity seems to be an attractive target of future investigation. Recently, however, elevated proinflammatory cytokines have been shown in mice with obesityrelated NASH. ${ }^{9}$ These increased cytokines are believed to downregulate insulin signaling with increased SOCS3 protein in the liver. ${ }^{32,33}$ Some other previous reports have also shown that long-term IL-6 exposure induced SOCS3 expression, caused insulin resistance and exacerbated liver injury, whereas short-term IL-6 exposure prevented hepatocyte apoptosis and steatosis after $\mathrm{CCl} 4$ treatment or partial hepatectomy. ${ }^{34,35}$ Hence, we hypothesized that continuous IL-6 stimulation induced liver inflammation and fibrosis.

An MCD diet is believed to induce accumulation of hepatic triglycerides by inhibiting mitochondrial $\beta$-oxidation of fatty acids and blocking hepatic export of VLDL. ${ }^{25}$ Unlike high-fat diets, which produce relatively little hepatic necroinflammation and fibrosis, MCD diets are used widely to induce NASH with fibrosis. Although an MCD diet inhibits mitochondrial oxidation of FA, thereby limiting that process as a source of ROS, this diet induces microsomal FA-oxidizing enzymes, such as Cyp2E1. The latter may produce sufficient ROS to trigger the secondary 'hits' that are required for progression from NAFLD to more advanced stages of fatty liver disease. ${ }^{36,37}$ Thus, MCD diets promote NASH with fibrosis, even in nonobese mice. Moreover, recent interesting reports have shown that not only decreased VLDL secretion but also increased fatty acid uptake and cytoplasmic fatty acid synthesis genes, despite decreased nuclear levels of lipogenic transcription factors, such as SREBP-1, are the major mechanisms by which the MCD diet promotes intrahepatic lipid accumulation. ${ }^{38}$ These discrepancies in lipogenic gene expression between the nuclei and cytoplasm seem to be present at an earlier stage of MCD diet-induced NASH. In this study, an MCD diet for 8 weeks decreased all lipogenic gene expression, mRNA levels of SREBPs, AcetylCoA Carboxylase, ATP-citrate lyase, fatty acid synthase and Stearoyl-CoA desaturase- 1 and protein levels of PPAR $\alpha$ in the liver (data not shown).

An MCD diet increased hepatic AMPK phosphorylation (Figure 1b). An MCD diet itself inhibits mitochondrial import of carnitine, thereby limiting FA oxidation that normally generates ATP. ROS/lipid peroxidation that results from alternative FA-oxidizing mechanisms also damages the mitochondria, further limiting ATP production. Gradual diet-related depletion of liver ATP pools activates AMPK. Furthermore, IL- 6 also increases AMPK rapidly and markedly. ${ }^{39,40}$ AMPK regulates the expression of genes involved in lipogenesis and mitochondrial biogenesis and also inhibits cell survival by binding to and phosphorylating insulin receptor substrate (IRS)-1 at Ser794. ${ }^{40}$ Phosphorylation of IRS-1 at this site inhibits phosphatidylinositol 3-kinase/Akt signaling, suppresses the mitochondrial membrane potential and promotes apoptosis. In this model, Akt signaling was considered to be inhibited by increased AMPK and STAT3induced SOCS3. ${ }^{11}$ Hence, it can be postulated that MR16-1 treatment contributed to the recovery of Akt activity, resulting in reduced hepatocyte apoptosis in this model.

In conclusion, MR16-1 treatment decreased SOCS3 expression successfully, suppressed STAT3 activation and improved plasma FFA elevation through increased fatty acid uptake and hepatic triglyceride biosynthesis, leading to decreased Cyp2E1 expression, ROS production and hepatic apoptosis. As shown by our previous study, hepatic triglyceride synthesis may have a compensatory role for an impaired mechanism of FFA detoxification. ${ }^{25}$ In clinical trial with neutralizing IL-6 receptor antibody, it was reported that plasma triglyceride and cholesterol levels were elevated in treated patients with rheumatoid arthritis. ${ }^{21,41}$ It is interesting and necessary to investigate whether hepatic steatosis in those patients is ameliorated or exacerbated. Hopefully, further experiments using MR16-1 in other NASH models will show the effect of neutralizing IL-6 receptor on the hepatic histology in NASH.

Supplementary Information accompanies the paper on the Laboratory Investigation website (http://www.laboratoryinvestigation.org)

\section{ACKNOWLEDGEMENT}

This study was supported by a Grant-in-Aid for Scientific Research from the Japan Society for the Program of Science (Kanji Yamaguchi).

\section{DISCLOSURE/CONFLICT OF INTEREST}

The authors declare no conflict of interest.

1. Ludwig J, Viggiano TR, McGill DB, et al. Nonalcoholic steatohepatitis: Mayo Clinic experience with a hitherto unnamed disease.. Mayo Clin Proc 1980;55:434-438.

2. Day CP, James OF. Steatohepatitis: a tale of two 'hit'? Gastroenterology 1998;114:842-845.

3. Baranova A, Schlauch $K$, Elariny $H$, et al. Gene expression patterns in hepatic tissue and visceral adipose tissue of patients with nonalcoholic fatty liver disease. Obes Surg 2007;17:1111-1118.

4. Jarrar MH, Baranova $A$, Collantes $R$, et al. Adipokines and cytokines in non-alcoholic fatty liver disease. Aliment Pharmacol Ther 2008;27:412-421.

5. Diehl AM. Tumor necrosis factor and its potential role in insulin resistance and nonalcoholic fatty liver disease. Clin Liver Dis 2004;8:619-638.

6. Cressman DE, Greenbaum LE, DeAngelis RA, et al. Liver failure and defective hepatocyte regeneration in interleukin-6-deficient mice. Science 1996;274:1379-1383.

7. Selzner $N$, Selzner $M$, Odermatt $B$, et al. ICAM-1 triggers liver regeneration through TNF and IL-6 release in mice. Gastroenterology 2003;124:692-700.

8. Anty R, Bekri S, Luciani N, et al. The inflammatory C-reactive protein is increased in both liver and adipose tissue in severely obese patients independently from metabolic syndrome, type 2 diabetes, and NASH. Am J Gastroenterol 2006;101:1824-1833.

9. Wieckowska A, Papouchado BG, Li Z, et al. Increased hepatic and circulating interleukin-6 levels in human nonalcoholic steatohepatitis. Am J Gastroenterol 2008;103:1372-1379.

10. Febbraio MA. Gp130 receptor ligands as potential therapeutic targets for obesity. J Clin Invest 2007;117:841-849.

11. Inoue $\mathrm{H}$, Ogawa $\mathrm{W}$, Ozaki $\mathrm{M}$, et al. Role of STAT-3 in regulation of hepatic gluconeogenic genes and carbohydrate metabolism in vivo. Nat Med 2004;10:168-174. 
12. Peters $M$, Blinn $G$, Jostock $T$, et al. Combined interleukin 6 and soluble interleukin 6 receptor accelerates murine liver regeneration. Gastroenterology 2000;119:1663-1671.

13. Klein C, Wüstefeld T, Assmus U, et al. The IL-6-gp130-STAT3 pathway in hepatocytes triggers liver protection in T cell-mediated liver injury. J Clin Invest 2005;115:860-869.

14. Kovalovich K, Li W, DeAngelis R, et al. Interleukin-6 protects against Fas-mediated death by establishing a critical level of anti-apoptotic hepatic proteins FLIP, Bcl-2, and Bcl-xl. J Biol Chem 2001;276: 26605-26613.

15. Sun Z, Klein AS, Radaeva $S$, et al. In vitro interleukin-6 treatment prevents mortality associated with fatty liver transplants in rats. Gastroenterology 2003;125:202-215.

16. Jin X, Zhang Z, Beer-Stolz D, et al. Interleukin-6 inhibits oxidative injury and necrosis after extreme liver resection. Hepatology 2007;46:802-812.

17. Ramsay AJ, Husband AJ, Ramshaw IA, et al. The role of interleukin- 6 in mucosal IgA antibody responses in vivo. Science 1994;264:561-563.

18. Selzner N, Selzner M, Jochum W, et al. Ischemic preconditioning protects the steatotic mouse liver against reperfusion injury: an ATP dependent mechanism. J Hepatol 2003;39:55-61.

19. Balkwill F, Mantovani A. Inflammation and cancer: back to Virchow? Lancet 2001;357:539-545.

20. Coussens LM, Werb Z. Inflammation and cancer. Nature 2002;420: 860-867.

21. Oldfield V, Dhillon S, Plosker GL. Tocilizumab: a review of its use in the management of rheumatoid arthritis. Drugs 2009;69:609-632.

22. Sahai A, Malladi $P$, Pan $X$, et al. Obese and diabetic $\mathrm{db} / \mathrm{db}$ mice develop marked liver fibrosis in a model of nonalcoholic steatohepatitis: role of short-form leptin receptors and osteopontin. Am J Physiol Gastrointest Liver Physiol 2004;287:G1035-G1043.

23. Okazaki $M$, Yamada $Y$, Nishimoto $N$, et al. Characterization of anti-mouse interleukin- 6 receptor antibody. Immunol Lett 2002;84:231-240.

24. Izumi-Nagai K, Nagai N, Ozawa Y, et al. Interleukin-6 receptor-mediated activation of signal transducer and activator of transcription-3 (STAT3) promotes choroidal neovascularization. Am J Pathol 2007;170: 2149-2158.

25. Yamaguchi K, Yang L, McCall S, et al. Inhibiting triglyceride synthesis improves hepatic steatosis but exacerbates liver damage and fibrosis in obese mice with nonalcoholic steatohepatitis. Hepatology 2007;45:1366-1374.

26. Anstee QM, Goldin RD. Mouse models in non-alcoholic fatty liver disease and steatohepatitis research. Int J Exp Pathol 2006;87:1-16.

27. Ota $\mathrm{T}$, Takamura $\mathrm{T}$, Kurita $\mathrm{S}$, et al. Insulin resistance accelerates a dietary rat model of nonalcoholic steatohepatitis. Gastroenterology 2007;132:282-293.
28. Uchiyama $\mathrm{Y}$, Yoshida $\mathrm{H}$, Koike $\mathrm{N}$, et al. Anti-IL-6 receptor antibody increases blood IL- 6 level via the blockade of IL- 6 clearance, but not via the induction of IL- 6 production. Int Immunopharmacol 2008;8:1595-1601.

29. Horiguchi N, Wang L, Mukhopadhyay P, et al. Cell type-dependent proand anti-inflammatory role of signal transducer and activator of transcription 3 in alcoholic liver injury. Gastroenterology 2008;134:1148-1158.

30. Brunt EM, Janney CG, Di Bisceglie AM, et al. Nonalcoholic steatohepatitis: a proposal for grading and staging the histological lesions. Am J Gastroenterol 1999;94:2467-2474.

31. Wallenius $\mathrm{V}$, Wallenius $\mathrm{K}$, Ahren $\mathrm{B}$, et al. Interleukin-6-deficient mice develop mature-onset obesity. Nat Med 2002;8:75-79.

32. Senn JJ, Klover PJ, Nowak IA, et al. Suppressor of cytokine signaling-3, a potential mediator of interleukin-6-dependent insulin resistance in hepatocytes. J Biol Chem 2003;278:13740-13746.

33. Ueki K, Kondo T, Tseng $\mathrm{YH}$, et al. Central role of suppressors of cytokine signaling proteins in hepatic steatosis, insulin resistance, and the metabolic syndrome in the mouse. Proc Natl Acad Sci 2004;101: 10422-10427.

34. Jin X, Zimmers TA, Perez EA, et al. Paradoxical effects of short- and long-term interleukin- 6 exposure on liver injury and repair. Hepatology 2006;43:474-484.

35. Rotter Sopasakis V, Larsson BM, Johansson A, et al. Short-term infusion of interleukin-6 does not induce insulin resistance in vivo or impair insulin signalling in rats. Diabetologia 2004;47:1879-1887.

36. Rinella ME, Elias MS, Smolak RR, et al. Mechanisms of hepatic steatosis in mice fed a lipogenic methionine choline-deficient diet. J Lipid Res 2008;49:1068-1076.

37. Leclercq IA, Farrell GC, Field J, et al. CYP2E1 and CYP4A as microsomal catalysts of lipid peroxides in murine nonalcoholic steatohepatitis. J Clin Invest 2000;105:1067-1075.

38. Larter CZ, Yeh MM, Haigh WG, et al. Hepatic free fatty acids accumulate in experimental steatohepatitis: role of adaptive pathways. J Hepatol 2008;48:638-647.

39. Ruderman NB, Keller C, Richard AM, et al. Interleukin-6 regulation of AMP-activated protein kinase. Potential role in the systemic response to exercise and prevention of the metabolic syndrome. Diabetes 2006;55(Suppl 2):S48-S54. Review.

40. Tzatsos A, Tsichlis PN. Energy depletion inhibits phosphatidylinositol 3-kinase/Akt signaling and induces apoptosis via AMP-activated protein kinase-dependent phosphorylation of IRS-1 at Ser-794. J Biol Chem 2007;282:18069-18082.

41. Hashizume M, Yoshida H, Koike N, et al. Over-produced IL-6 decreases blood lipid levels via up-regulation of VLDLR. Ann Rheum Dis 2010;69:741-746. 